\section{$\beta$-Agonists and asthma: too much of a good thing?}

\author{
Stephanie A. Shore ${ }^{1}$ and Jeffrey M. Drazen ${ }^{1,2}$ \\ ${ }^{1}$ Physiology Program, Harvard School of Public Health, and \\ 2Department of Medicine, Brigham and Women's Hospital, Boston, \\ Massachusetts, USA
}

In an unusual paradox, asthmatics who are chronically treated with bronchodilating $\beta$-agonists sometimes experience a worsening of their condition. A new study (see the related article beginning on page 619) describes one possible mechanism and reveals a potential new therapeutic target in the treatment of asthma.

J. Clin. Invest. 112:495-497 (2003). doi:10.1172/JCI200319642.

Inhaled selective $\beta_{2}$-agonists are the most widely used treatment for the acute relief of asthma symptoms. In patients with asthma, these agents cause bronchodilation (improvement in lung mechanics) and bronchoprotection (reduced responsiveness to nonspecific contractile stimuli). These actions result from binding to the $\beta_{2}$-adrenergic receptor $\left(\beta_{2} \mathrm{AR}\right)$, a heptahelical receptor that couples predominantly to the stimulatory $G$ protein, $G_{s}$ (Figure 1a). Once activated by receptor-ligand binding, the $\alpha$ subunit of $\mathrm{G}_{\mathrm{s}}$ activates adenylyl cyclase (AC), resulting in cAMP formation. cAMP phosphorylates protein kinase $\mathrm{A}$ (PKA), which phosphorylates multiple proteins, leading to reductions in intracellular calcium, smooth muscle relaxation, and bronchodilation.

It is possible to have too much of a good thing. Despite the ability of $\beta$-agonists to effect immediate reversal of airway obstruction, there has been continuing concern that regu-

\footnotetext{
Address correspondence to: Stephanie Shore, Physiology Program, Harvard School of Public Health, 665 Huntington Avenue, Boston, Massachusetts 02115, USA. Phone: (617) 432-0199; Fax: (617) 432-3468; E-mail: sshore@hsph.harvard.edu. Conflict of interest: The authors have declared that no conflict of interest exists. Nonstandard abbreviations used: $\beta_{2}$-adrenergic receptor $\left(\beta_{2} \mathrm{AR}\right)$; protein kinase A (PKA); phospholipase C- $\beta$ (PLC $\beta$ ); inositol 1,4,5 trisphosphate $\left(\mathrm{IP}_{3}\right)$; extracellular signal-regulated kinase (ERK); $G$ protein receptor kinase (GRK).
}

lar use of these drugs may be associated with adverse outcomes. In some, but not all, studies, regularly scheduled use (e.g., multiple times per day, every day) of inhaled $\beta$-agonists has resulted in loss of asthma

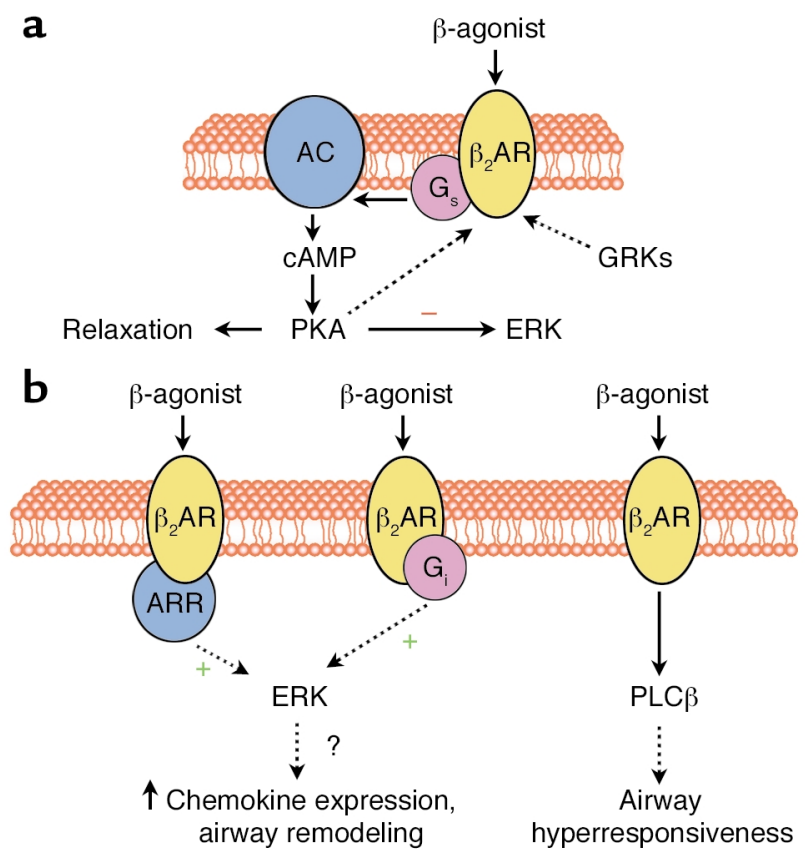

Figure 1

(a) Mechanism of $\beta$-agonist-induced airway smooth muscle relaxation. Ligand binding to the $\beta_{2} A R$ activates $G_{s}$, leading to adenylyl cyclase (AC) activation, cAMP formation, and subsequent protein kinase $A$ (PKA) activation. PKA phosphorylation of target proteins leads to smooth muscle relaxation and may also inhibit ERK activation. PKA also phosphorylates the $\beta_{2} A R$, leading to increased $G_{i}$ coupling. In addition, ligand binding causes $G$ protein receptor kinase (GRK) phosphorylation of the $\beta_{2} A R$, recruiting $\beta$-arrestin (ARR). (b) Inflammatory events in the asthmatic airway or regular $\beta$-agonist use may augment $G$ coupling and/or increase $\beta$-arrestin binding. Under these circumstances, $\beta$-agonists may result in ERK activation, potentially amplifying production of inflammatory cytokines and leading to airway remodeling. Persistent activation of the $\beta_{2} A R$ may also lead to phospholipase $C-\beta$ (PLC $\beta$ ) expression and consequent airway hyperresponsiveness (8). 
acting on receptors that couple to $\mathrm{G}_{\mathrm{q}}$ and activate PLC $\beta$, chronic $\beta$-agonists might augment the effects of these bronchoconstrictors. How could this occur? PLC $\beta$ hydrolyzes phosphatidylinositol 4,5 biphosphate, resulting in inositol $1,4,5$ trisphosphate $\left(\mathrm{IP}_{3}\right)$ production. $\mathrm{IP}_{3}$ increases intracellular calcium, leading to activation of myosin light chain kinase, myosin phosphorylation, and consequent muscle contraction. If the observations of McGraw et al. are borne out, this mechanism could explain the adverse effects of chronic stimulation of the $\beta_{2} \mathrm{AR}$. However, before we embrace this hypothesis, which is based on data from mouse airways, it will be important to confirm the observations of McGraw et al. in human airway smooth muscle stimulated with $\beta$-agonists rather than by $\beta_{2} \mathrm{AR}$ overexpression.

We think that there may be additional explanations for the bronchoconstrictor effects of chronic $\beta_{2} \mathrm{AR}$ stimulation. For example, in some cell types the $\beta_{2} \mathrm{AR}$ can couple to $G_{i}$ as well as $G_{s}(9-12)$. The switch from $G_{s}$ to $G_{i}$ coupling appears to be regulated by PKA phosphorylation of the $\beta_{2} A R$ (13). $\beta_{2} A R$-induced $G_{i}$ activation leads to activation of the extracellular signal-regulated kinase (ERK) MAPKs (Figure 1b) through a pathway dependent on the $G_{i} \beta \gamma$ subunits and activation of Ras (12). $\beta_{2} \mathrm{AR}$ activation also leads to $G$ protein receptor kinase-induced (GRK-induced) phosphorylation of the $\beta_{2} \mathrm{AR}$, resulting in its interaction with $\beta$ arrestin. $\beta$-arrestin can serve as a scaffolding protein linking the $\beta_{2} \mathrm{AR}$ to both the ERK and JNK MAPK pathways $(9,14)$. To complicate the matter, $\mathrm{G}_{\mathrm{s}}$-dependent formation of cAMP by $\beta_{2}$ AR activation can also inhibit ERK activation (10), apparently as a result of Raf- 1 phosphorylation by PKA (15). The ultimate effect of $\beta_{2} \mathrm{AR}$ activation on ERK phosphorylation is likely to reflect a balance of these various events.

Although these pathways have been described in other cell types, none of these events has been examined in airway smooth muscle cells. Thus it is interesting to consider the potential functional implications of $\beta$-agonist-induced ERK activation in these cells. Persistent ERK activation is known to be required for mitogenesis in airway smooth muscle cells (16). Activation of ERK is also required for the full expression by airway smooth muscle of the eosinophil chemotactic factors eotaxin and RANTES, as well as other chemokines (17). $\beta$-agonists normally inhibit mitogenesis (18) and inhibit expression of eotaxin (19) in airway smooth muscle. However, there may be conditions in which this is not the case. For example, $G_{s}$-to- $G_{i}$ switching could be exaggerated by inflammatory cytokines, which have been shown to increase $G_{i}$ expression (20). Inflammatory cytokines have also been shown to increase GRK expression in these cells (20). Increased GRK activation could be expected to enhance ERK activation through effects on $\beta$-arrestin binding to the $\beta_{2} \mathrm{AR}$. It is also possible that the balance of ERK-activating and ERKinhibiting effects of $\beta_{2} \mathrm{AR}$ activation may be affected by $\beta_{2} A R$ desensitization under conditions of regular $\beta$-agonist use.

It is interesting to note that the adverse effects of regular $\beta$-agonist use can be observed even weeks after their withdrawal, at a time when $\beta_{2} \mathrm{AR}$ desensitization should be resolved (2). If regular use of $\beta$-agonists negatively impacts the balance of factors contributing to airway smooth muscle mitogenesis or airway remodeling in asthma, it could have long-term consequences. Importantly, studies of regular use of $\beta$-agonist have reported adverse effects only in subjects homozygous for the Arg16 variant of the $\beta_{2} \mathrm{AR}(2,3,5)$. The impact of $\beta_{2} \mathrm{AR}$ polymorphisms on non- $\mathrm{G}_{\mathrm{s}}$-mediated events such as ERK phosphorylation has not been examined in any cell type.

$\beta$-agonists are currently one of the most important forms of therapy for asthma. Little is known about non- $G_{s}$-mediated effects of $\beta_{2} A R$ activation and other events that could negatively impact the function of airway smooth muscle in asthma. In this issue of the JCI,
McGraw et al. (8) report one such event, induction of PLC $\beta$, but it is possible that this is just the tip of the iceberg. Understanding the panoply of $\beta_{2}$ AR-mediated events in airway smooth muscle might lead to the design of new agonists that avoid negative effects of $\beta_{2} A R$ activation while enhancing events that lead to relaxation.

1. Vathenen, A.S., Knox, A.J., Higgins, B.G., Britton, J.R., and Tattersfield, A.E. 1988. Rebound increase in bronchial responsiveness after treatment with inhaled terbutaline. Lancet. 1:554-558.

2. Israel, E., et al. 2000. The effect of polymorphisms of the beta(2)-adrenergic receptor on the response to regular use of albuterol in asthma. Am. J. Respir. Crit. Care Med. 162:75-80.

3. Taylor, D.R., et al. 2000. Asthma exacerbations during long term beta agonist use: influence of beta(2) adrenoceptor polymorphism. Thorax. 55:762-767.

4. Taylor, D.R., et al. 1998. Asthma control during long-term treatment with regular inhaled salbutamol and salmeterol. Thorax. 53:744-752.

5. Hancox, R.J., Sears, M.R., and Taylor, D.R. 1998 Polymorphism of the beta2-adrenoceptor and the response to long-term beta2-agonist therapy in asthma. Eur. Respir. J. 11:589-593.

6. Sears, M.R., et al. 1990. Regular inhaled betaagonist treatment in bronchial asthma. Lancet. 336:1391-1396.

7. Hanania, N.A., Sharafkhaneh, A., Barber, R., and Dickey, B.F. 2002. Beta-agonist intrinsic efficacy: measurement and clinical significance. Am. J. Respir. Crit. Care Med. 165:1353-1358.

8. McGraw, D.W., Almoosa, K.F., Paul, R.J., Kobilka, B.K., and Liggett, S.B. 2003. Antithetic regulation by $\beta$-adrenergic receptors of $G_{q}$ receptor signaling via phospholipase $C$ underlies the airway $\beta$-agonist paradox. J. Clin. Invest. 112:619-626. doi:10.1172/JCI200318193.

9. Tohgo, A., et al. 2003. The stability of the G protein-coupled receptor-beta-arrestin interaction determines the mechanism and functional consequence of ERK activation. J. Biol. Chem. 278:6258-6267.

10. Crespo, P., Cachero, T.G., Xu, N., and Gutkind, J.S. 1995. Dual effect of beta-adrenergic receptors on mitogen-activated protein kinase. Evidence for a beta gamma-dependent activation and a $G$ alpha s-cAMP-mediated inhibition. J. Biol. Chem. 270:25259-25265.

11. Zhu, W.Z., et al. 2001. Dual modulation of cell survival and cell death by beta(2)-adrenergic signaling in adult mouse cardiac myocytes. Proc. Natl. Acad. Sci. U. S. A. 98:1607-1612.

12. Zou, Y., et al. 1999. Both Gs and Gi proteins are critically involved in isoproterenolinduced cardiomyocyte hypertrophy. J. Biol. Chem. 274:9760-9770.

13. Daaka, Y., Luttrell, L.M., and Lefkowitz, R.J. 1997. Switching of the coupling of the beta2 adrenergic receptor to different $G$ proteins by protein kinase A. Nature. 390:88-91.

14. Hall, R.A., and Lefkowitz, R.J. 2002. Regulation of $\mathrm{G}$ protein-coupled receptor signaling by scaffold proteins. Circ. Res. 91:672-680.

15. Cook, S.J., and McCormick, F. 1993. Inhibition by cAMP of Ras-dependent activation of Raf. Science. 262:1069-1072.

16. Orsini, M.J., et al. 1999. MAPK superfamily activation in human airway smooth muscle: mitogenesis requires prolonged $\mathrm{p} 42 / \mathrm{p} 44$ activation. Am. J. Physiol. 277:L479-L488.

17. Hallsworth, M.P., Moir, L.M., Lai, D., and Hirst, S.J. 2001. Inhibitors of mitogen-activated pro- 
tein kinases differentially regulate eosinophilactivating cytokine release from human airway smooth muscle. Am. J. Respir. Crit. Care Med. 164:688-697.

18. Roth, M., et al. 2002. Interaction between glucocorticoids and beta2 agonists on bronchial airway smooth muscle cells through synchronised cellular signalling. Lancet. 360:1293-1299.

19. Pang, L., and Knox, A.J. 2001. Regulation of TNFalpha-induced eotaxin release from cultured human airway smooth muscle cells by beta2-agonists and corticosteroids. FASEB J. 15:261-269.
20. Mak, J.C., Hisada, T., Salmon, M., Barnes, P.J., and Chung, K.F. 2002. Glucocorticoids reverse IL-1beta-induced impairment of beta-adrenoceptor-mediated relaxation and up-regulation of G-protein-coupled receptor kinases. $\mathrm{Br} . \mathrm{J}$. Pharmacol. 135:987-996.

\section{Thyroid hormone action: a binding contract}

\author{
Mitchell A. Lazar
}

Division of Endocrinology, Diabetes, and Metabolism, Departments of Medicine and Genetics, and The Penn Diabetes Center, University of Pennsylvania School of Medicine, Philadelphia, Pennsylvania, USA

Thyroid hormones are critical for differentiation, growth, and metabolism. A new study (see the related article beginning on page 588) investigating the biological role of the TH receptor TR- $\beta$ has demonstrated that DNA binding is critical for most of its functions, but also suggests that novel mechanisms independent of DNA binding may contribute to regulation of auditory function by TR- $\beta$.

J. Clin. Invest. 112:497-499 (2003). doi:10.1172/JCI200319479.
Thyroid hormone $(\mathrm{TH})$ plays a critical role in the development and adult functions of many organs and tissues. Many of the effects of TH are mediated by a family of high-affinity receptor proteins, called $\mathrm{TH}$ receptors (TRs). Three functional TRs, TR- $\beta 1$, TR- $\beta 2$, and TR- $\alpha 1$, are encoded on two mammalian genes (1). The TR- $\alpha$ gene also encodes variant proteins that do not bind $\mathrm{TH}$ and whose function may be to inhibit the action of other TRs (2). The TRs are members of a larger family of nuclear receptors (NRs) for lipophilic signaling molecules that includes steroid hormones, vitamin derivatives such as retinoic acid and vitamin $\mathrm{D}_{3}$, fatty acid and cholesterol

\footnotetext{
Address correspondence to: Mitchell A. Lazar, University of Pennsylvania School of Medicine, 611 Clinical Research Building, 415 Curie Boulevard, Philadelphia, Pennsylvania 19104-6149, USA. Phone: (215) 898-0198; Fax: (215) 898-5408; E-mail: lazar@mail.med.upenn.edu. Conflict of interest: The author has declared that no conflict of interest exists.

Nonstandard abbreviations used: thyroid hormone (TH); $\mathrm{TH}$ receptor (TR); nuclear receptor (NR); retinoid X receptor (RXR); thyroid hormone response element (TRE); NR corepressor (N-CoR); silencing mediator of retinoid and thyroid receptors (SMRT); histone deacetylase (HDAC); thyroid-stimulating hormone (TSH); negative TRE (nTRE).
}

metabolites, and xenobiotics (3). As the family name suggests, a key aspect of TR function involves nuclear regulation of gene transcription. In this issue of the JCI, Shibusawa and colleagues use mice whose TR- $\beta$ gene products cannot bind DNA in order to shed light on the mechanisms by which TR functions in development and physiology (4). The TR- $\beta$ mutant mice have a phenotype that is similar but not identical to that of mice lacking TR- $\beta$, suggesting that most but not all TR functions involve direct DNA binding.

\section{Positive regulation of gene expression by $\mathrm{TH}$ : a simple model}

Like nearly all NRs, the central portion of TR contains a zinc-ordered domain that binds to double-stranded DNA

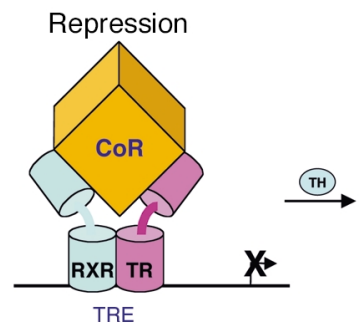

with a well-characterized sequence specificity. TR recognizes the sequence AGGTCA, to which it can bind as a monomer. This sequence also serves as a half-site for TR homodimers as well as heterodimers with the retinoid $X$ receptor (RXR) (5). RXR increases the DNA-binding affinity of TR and also restricts binding to a subset of half-site arrangements whose binding by the TR/RXR heterodimer is energetically favorable. The most stable binding occurs on the classical DR4 thyroid response element (TRE), in which two halfsites are directly repeated with a spacing of $4 \mathrm{bp}$ (6). The structural basis of this binding preference has been determined (7).

On a TRE-containing target gene, $\mathrm{TH}$ binding acts as a switch between repressed and activated states (Figure 1) (8). Genes that are bound by TR/RXR heterodimers, and potentially TR homodimers, are actively repressed in the absence of $\mathrm{TH}$. The repression function is located in the C-terminal ligand-binding domain, which binds to the corepressor molecules nuclear receptor corepressor (N-CoR) and silencing mediator of retinoid and thyroid receptors (SMRT) (9), which anchor large multiprotein complexes containing histone deacetylase (HDAC) activity and mediate ligand-independent repression. $\mathrm{TH}$ binding induces a conformational change that destabilizes corepressor binding and that favors binding of transcriptional coactiva-

.

